\title{
TASA DE CESÁREAS POR GRUPOS DE ROBSON EN UNA INSTITUCIÓN DE MEDIANA COMPLEJIDAD DE LA CIUDAD DE BOGOTÁ,
} 2012-2014

\author{
Cesarean section rate by Robson groups in an \\ intermediate complexity centre in the city of \\ Bogotá, 2012-2014
}

Diana Liliana Jiménez-Hernández, $M D^{1}$; Andrea del Pilar Guevara-Rodríguez, $M D^{1}$; John Jairo Zuleta-Tobón, MD, $\mathrm{MSc}^{2}$; Jorge Andrés Rubio-Romero, $\mathrm{MD}, \mathrm{MSc}^{3}$

Recibido: febrero 26/16 - Aceptado: junio 13/16

\section{RESUMEN}

Introducción: la clasificación de Robson permite caracterizar las mujeres a las que se les realiza cesárea para implementar estrategias a fin de racionalizar este recurso. El objetivo fue determinar las proporciones específicas de cesárea según la clasificación de Robson en una institución hospitalaria de mediana complejidad.

Materiales y métodos: estudio descriptivo transversal que incluyó mujeres atendidas en un hospital público de atención general, que recibe pacientes del aseguramiento financiado por el Estado, entre 2012 y 2014. Se llevó a cabo muestreo aleatorio estratificado según la vía del parto, basado en la razón parto:cesárea encontrada en el periodo, clasificando las mujeres atendidas en los 10 grupos de Robson. Se realizó el análisis descriptivo de las variables demográficas, indicaciones clínicas reportadas y las proporciones global y específica de uso de cesárea

1 Médico residente, Departamento de Obstetricia y Ginecología, Universidad Nacional de Colombia, Bogotá (Colombia).

2 Profesor titular, Departamento de Obstetricia y Ginecología, Universidad de Antioquia, Medellín (Colombia).

3 Profesor titular, Departamento de Obstetricia y Ginecología, Universidad Nacional de Colombia, Bogotá (Colombia). jarubior@unal.edu.co en cada grupo. El estudio contó con aprobación del comité de ética institucional.

Resultados: se seleccionaron 1.190 de 6.558 nacimientos ocurridos durante el periodo. La proporción global de cesárea fue de 37,0\%. Las mujeres nulíparas contribuyeron con el 38,5\% de las cesáreas, seguidas por el grupo con cesárea anterior (36,4\%) que tuvo una proporción específica del $100 \%$. La proporción específica de cesárea en el grupo de mujeres nulíparas en trabajo de parto espontáneo fue 36,6\% y la del grupo de partos prematuros 43,3\%.

Conclusiones: las mujeres con cesárea anterior, con embarazo pretérmino y las nulíparas en parto espontáneo o inducido tienen las proporciones específicas más elevadas de cesárea. Estos grupos podrían ser susceptibles de intervención para impactar las tasas de cesárea de la institución.

Palabras clave: cesárea, parto vaginal después de cesárea, cesárea repetida, clasificación, prevalencia.

\section{ABSTRACT}

Introduction: The Robson classification is useful for characterizing women taken to Cesarean section with the aim of implementing successful cesarean 
section rate reduction strategies. The objective was to determine specific Cesarean section proportions according to the Robson classification in an intermediate complexity hospital.

Materials and methods: cross-sectional descriptive study that included women of the state-subsidized health insurance regime seen at a general public hospital between 2012 and 2014. A stratified random sampling was performed on the basis of the birth route, per delivery, based on the delivery/Cesarean section ratio found during the time period. The women were classified under 10 Robson groups. A descriptive analysis was conducted of the demographic variables, the clinical indications reported, and the overall and specific proportions of Cesarean sections in each group. The study was approved by the Ethics Committee of the Institution.

Results: Out of 6558 deliveries during the study period, 1190 were selected. The overall proportion of Cesarean sections was $37.0 \%$. Nulliparous women accounted for $38.5 \%$ of the Cesarean sections, followed by the group with previous Cesarean sections (36.4\%) for which the specific proportion was $100 \%$. The specific proportion of Cesarean sections in nulliparous women in spontaneous labour was $36.6 \%$, whereas it was $43.3 \%$ in the premature delivery group.

Conclusions: The highest specific proportions of Cesarean section were found among women with a previous Cesarean section, women with pre-term delivery and nulliparous women with spontaneous or induced delivery. These groups could lend themselves for intervention in order to have an impact on the rates of Cesarean section in the Institution.

Key words: Cesarean section, vaginal delivery after Cesarean section, repeated Cesarean section, Classification, prevalence.

\section{INTRODUCCIÓN}

En las últimas décadas se ha presentado un aumento progresivo en la proporción de cesáreas en la mayo- ría de países, con incrementos de hasta un 70 \% (1). Colombia no es ajena a este problema, pues según la información del Observatorio de Salud Materna de Colombia (OMAT), reportada en el Sistema Integral de Información del Ministerio de Salud y Protección Social, se pasó de una proporción de cesáreas del 31,3\% para el año 2005 a un $46 \%$ para el año 2014 (2). Este aumento en la frecuencia no puede ser explicado completamente por cambios en las indicaciones médicas del procedimiento (3).

La Organización Mundial de la Salud (OMS) sostiene que no existen beneficios adicionales con proporciones de cesárea por encima del $15 \%(4,5)$. El aumento en la tasa de cesáreas por encima de lo recomendado por la OMS conlleva un aumento en las complicaciones maternas y perinatales: el "Estudio mundial de la OMS 2005 sobre salud materna y perinatal", realizado en 120 instituciones de Latinoamérica, encontró que a mayor proporción de cesáreas se observa un incremento en el riesgo de mortalidad materna, morbilidad materna grave (transfusión sanguínea, histerectomía posparto, ingreso a unidad de cuidados intensivos, muerte materna y estancia hospitalaria mayor de 7 días) y tratamiento posnatal materno con antibióticos (6). Un estudio similar realizado en Asia encontró que los riesgos asociados a la cesárea se mantienen incluso cuando se realiza cesárea electiva en pacientes sin comorbilidades que expliquen algunas de las complicaciones y se incrementan cuando se realiza cesárea de urgencia, siendo este grupo el que aporta la mayor proporción de la mortalidad $(7,8)$. Simultáneamente, la cesárea incrementa los riesgos para los recién nacidos evidenciados por mayor número de muertes fetales, neonatales y mayor número de recién nacidos con necesidad de ingreso a unidades de cuidado intensivo por siete o más días (7-11).

Ante la realidad de que la proporción de cesáreas es excesiva, según los parámetros definidos por la OMS, se han propuesto diferentes sistemas de clasificación para realizar diagnóstico y vigilancia de esta tendencia, y para proponer intervenciones en 
grupos específicos con la intención de disminuir la frecuencia de la operación cesárea (12-15)

El sistema propuesto en 2001 por Robson et al. (15) define diez grupos con base en cuatro conceptos obstétricos básicos: categoría del embarazo, antecedentes obstétricos, curso de trabajo de parto y edad gestacional. Estos parámetros son evaluados de forma prospectiva y permiten comparaciones en el tiempo para una misma unidad asistencial y entre distintas entidades. La clasificación objetiva acorde con las características clínicas observadas, identifica aquellos grupos que contribuyen en mayor medida a la tasas de cesárea y facilita el reconocimiento de aquellos susceptibles de intervención (15-17).

El modelo de Robson ha sido implementado y ha mostrado amplia versatilidad y adaptabilidad en diferentes hospitales y regiones del mundo (18-30). En 2014, la Federación Colombiana de Obstetricia y Ginecología y la Federación Colombiana de Perinatología propusieron el uso del modelo de Robson como un instrumento útil para analizar la proporción de cesáreas en cada institución y en el país, conocer el impacto de esta intervención sobre la morbilidad materna y neonatal, proponer estrategias para disminuir de manera segura la frecuencia de parto por cesárea e identificar las tendencias con respecto a sus indicaciones. Por tanto, y dentro de este marco, es importante establecer una línea de base que permita comparaciones en el tiempo en las instituciones que atienden partos.

El objetivo del presente estudio fue evaluar la utilización de la cesárea como vía del parto en una institución pública de mediano nivel de complejidad y de carácter universitario mediante la implementación del modelo de Robson, e identificar los grupos más proclives a ser llevados a este procedimiento.

\section{MATERIALES Y MÉTODOS}

Estudio descriptivo, de corte transversal, llevado a cabo en un hospital público de mediano y alto nivel de complejidad, de la ciudad de Bogotá, que atiende pacientes de bajo y alto riesgo obstétrico, pertenecientes al aseguramiento financiado por el
Estado en el sistema general de seguridad social en Colombia, donde se realizan prácticas clínicas de programas de formación en pre y posgrado de medicina.

Se incluyeron todas las mujeres ingresadas para atención de parto de fetos mayores de $500 \mathrm{~g}$, entre los años 2012 a 2014. Del total de nacimientos del Hospital de Engativá ocurridos en el periodo, se definió una muestra aleatoria del $18 \%$ de los nacimientos ocurridos en la institución durante el periodo de estudio y se contempló un $10 \%$ adicional por datos faltantes con el fin de garantizar la presencia de pacientes en cada uno de los grupos de Robson; se realizó muestreo aleatorio estratificado por afijación proporcional basado en la razón de parto: cesárea encontrada durante el periodo de estudio de las mujeres que cumplieron con los criterios de inclusión. Se definieron como criterios de exclusión las pacientes con atención de parto extra hospitalario o sin información para clasificarlas en el grupo de Robson correspondiente.

Procedimiento. Recolección de datos y control de calidad: se diseñó un instrumento de recolección de datos con las variables necesarias para clasificar los nacimientos en los 10 grupos de Robson, para lo cual se realizó un entrenamiento y una prueba piloto a fin de identificar las barreras y ajustar el instrumento de recolección. El total de nacimientos durante el periodo de estudio y la vía del parto se obtuvieron de los egresos hospitalarios, a partir de los cuales se realizó el muestreo aleatorio y la obtención de las historias clínicas para la recolección de los datos y la clasificación de las mujeres en los grupos de Robson en formatos físicos, por dos investigadores de manera independiente y luego digitalizada en una base de datos electrónica creada en Excel. Se hizo una segunda revisión de los datos y validación de la base de datos mediante revisión aleatoria de la información y su consistencia con los instrumentos de recolección y las historias clínicas.

Definición de variables. Para el estudio se registraron características demográficas tales como edad materna (años cumplidos al momento del naci- 
miento), estado civil, escolaridad, seguridad social (subsidiado, contributivo, vinculado/no asegurado, especial, excepción) y procedencia. También se registró la información necesaria para clasificar a las mujeres dentro de los 10 grupos de Robson, paridad (nulípara, multípara), número de gestaciones y edad gestacional al momento del parto (mayor o igual a 37 semanas, < 37 semanas); presentación (cefálica, podálica/pélvica, transversa/oblicua); multiplicidad de la gestación (embarazo único, embarazo múltiple); inicio del trabajo de parto (espontáneo, inducido) y antecedente de cesárea. Se definieron las indicaciones de cesárea y se agruparon teniendo en cuenta su origen (materna, fetal) y el diagnóstico de indicación de cesárea. La proporción de global y específica en cada grupo de Robson se estimó como el número de nacimientos por cesárea sobre el número total de nacimientos ocurrido en el periodo de observación (19).

Análisis estadístico. Los datos obtenidos se analizaron en Epi-Info® v 7.0 y Stata $\circledR_{\text {v 10.0. Se realizó }}$ descripción de las variables nominales cualitativas con frecuencias absolutas y relativas. Para las variables de tipo cuantitativo se realizó análisis descriptivo con estadísticos de tendencia central y dispersión según las pruebas de normalidad de las variables.

Aspectos éticos. Se mantuvo la confidencialidad de la información y se respetaron los derechos de las mujeres participantes, el estudio fue aprobado por el Comité de ética de la Facultad de Medicina de la Universidad Nacional (Acta 005-034-15) y el Comité de investigaciones del Hospital de Engativá (Acta 004-2015).

\section{RESULTADOS}

En el Hospital de Engativá se atendieron un total de 6.558 nacimientos durante el periodo comprendido entre 2012 y 2014, con una proporción global de 37,0 \% cesáreas y 63,0 \% partos vaginales. Se obtuvo una muestra aleatoria estratificada por vía del parto de 1.190 pacientes teniendo en cuenta lla razón de partos:cesáreas ocurridos, como se muestra en la figura 1.
Figura 1. Flujograma de pacientes

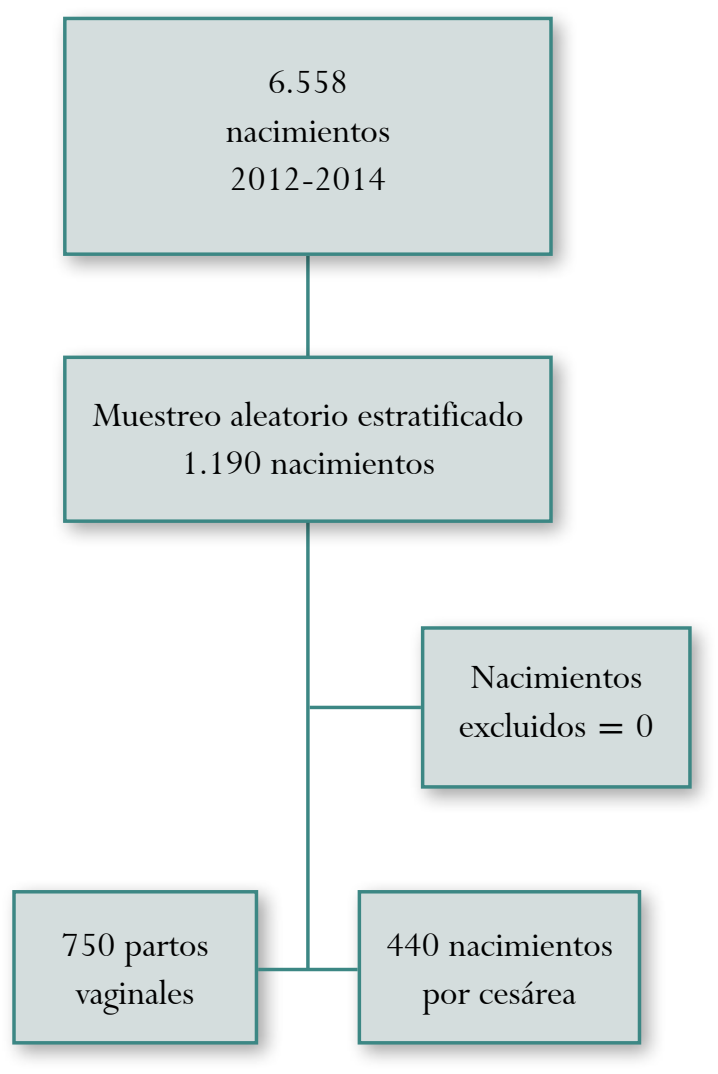

La edad promedio de las mujeres atendidas fue de 22,8 años; el 57,7\% correspondió a mujeres en su primera gestación, el 9,8 \% tuvo gestaciones pretérmino y el $3 \%$ presentaciones fetales diferentes a la cefálica (tabla 1). El peso promedio de los neonatos nacidos por cesárea fue de $2.943 \mathrm{~g}$, con una desviación estándar de 527 g, un peso mínimo de 500 g y un peso máximo de 4.500 g; el promedio de talla fue 49,5 cm, con una desviación estándar de $2,95 \mathrm{~cm}$, un valor mínimo de $21 \mathrm{~cm}$ y valor máximo de $57 \mathrm{~cm}$. La distribución de las pacientes incluidas dentro de los 10 grupos de Robson, la proporción de cesáreas en cada uno de ellos y su contribución global a la proporción de cesáreas realizadas en la institución se observan en la tabla 2. El grupo de Robson más numeroso correspondió al de lasmujeres nulíparas a quienes se realizó inducción o cesárea antes del inicio del trabajo de parto (grupo 2), constituido por 405 mujeres. Los grupos que 


\begin{tabular}{|c|c|c|}
\hline Variable & Característica & n (\%) \\
\hline Edad materna & $\begin{array}{l}\text { Promedio (DE): } \\
\text { Mínima-Máxima }\end{array}$ & $\begin{array}{c}22,8(5,8) \\
12-45\end{array}$ \\
\hline Estado civil & $\begin{array}{l}\text { Soltera } \\
\text { Casada } \\
\text { Viuda } \\
\text { Separada/divorciada } \\
\text { Unión libre } \\
\text { Sin dato }\end{array}$ & $\begin{array}{c}400(33,6) \\
120(10,1) \\
64(5,4) \\
4(0,3) \\
601(50,5) \\
1(0,1)\end{array}$ \\
\hline Escolaridad & $\begin{array}{l}\text { Primaria } \\
\text { Bachillerato } \\
\text { Técnico } \\
\text { Tecnólogo } \\
\text { Profesional } \\
\text { Posgrado } \\
\text { Sin estudio }\end{array}$ & $\begin{array}{c}83(7,0) \\
968(81,3) \\
61(5,1) \\
18(1,5) \\
56(4,7) \\
2(0,2) \\
2(0,2)\end{array}$ \\
\hline Tipo de seguridad social & $\begin{array}{l}\text { Subsidiado } \\
\text { Contributivo } \\
\text { Vinculado/no asegurado } \\
\text { Especial } \\
\text { Excepción }\end{array}$ & $\begin{array}{c}669(56,2) \\
46(3,8) \\
475(39,9) \\
0(0,0) \\
0(0,0)\end{array}$ \\
\hline Procedencia & $\begin{array}{l}\text { Bogotá rural } \\
\text { Bogotá urbana } \\
\text { Fuera de Bogotá urbano } \\
\text { Fuera de Bogotá rural }\end{array}$ & $\begin{array}{c}171(14,3) \\
992(83,3) \\
18(1,5) \\
8(0,6)\end{array}$ \\
\hline Paridad & $\begin{array}{l}\text { Nulípara - Primera gestación } \\
\text { Multípara } 2 \text { o más }\end{array}$ & $\begin{array}{l}687(57,7) \\
503(42,2)\end{array}$ \\
\hline Edad gestacional & $\begin{array}{l}\text { Mayor o igual a } 37 \text { semanas } \\
\text { Menor } 37 \text { semanas }\end{array}$ & $\begin{array}{c}1073(90,2) \\
117(9,8)\end{array}$ \\
\hline
\end{tabular}

más aportaron a la proporción de cesáreas fueron las mujeres nulíparas con el 38,5\% (21,4\% con inducción del trabajo de parto o cesárea sin trabajo de parto, y $17 \%$ en trabajo de parto espontáneo que tuvieron proporciones específicas de cesárea de 23,3 y 36,6\%, respectivamente), seguido del grupo de mujeres con embarazo a término en presentación cefálica y cesárea previa con el 36,4\% (grupo 5). Este último, junto con los grupos 6 al 9, tuvieron una proporción específica de cesárea del 100 \%. Las mujeres con parto prematuro (grupo 10) tuvieron una proporción específica del 43,3 \% y las nulíparas en trabajo de parto espontáneo 36,6\% (tabla 2). Las tres principales indicaciones registradas en las historias clínicas para la realización de cesárea fueron cesárea previa con un 27,7\%, inducción fallida con un $18,2 \%$ y sospecha de estado fetal insatisfactorio con un $10,7 \%$ (tabla 3).

\section{DISCUSIÓN}

En el Hospital de Engativá se encontró una proporción global de cesáreas de 37,0 \% en los años 2012 a 2014, inferior a la proporción de parto por cesárea de $42 \%$ calculada para Bogotá en 2014 y dentro de 


\begin{tabular}{|c|c|c|c|}
\hline \multicolumn{4}{|c|}{$\begin{array}{l}\text { Tabla } 2 . \\
\text { Distribución y tasa de cesárea por grupos de Robson en el } \\
\text { Hospital de Engativá, Bogotá (Colombia), 2012-2014 }\end{array}$} \\
\hline Grupos & $\begin{array}{l}\text { Tamaño relativo } \\
\text { de los grupos } \\
\mathbf{N}(\%)\end{array}$ & $\begin{array}{l}\text { Proporción específica } \\
\text { de nacimientos por } \\
\text { cesárea en cada grupo } \\
\text { N (\%) }\end{array}$ & $\begin{array}{l}\text { Contribución hecha por } \\
\text { cada grupo al porcentaje } \\
\text { general de cesáreas } \\
\text { N }(\%)\end{array}$ \\
\hline $\begin{array}{l}\text { Mujeres nulíparas con embarazo simple } \\
\text { en cefálica mayor o igual a } 37 \text { semanas, } \\
\text { en trabajo de parto espontáneo. }\end{array}$ & $205(17,2)$ & $75 / 205(36,6)$ & $75 / 440(17,1)$ \\
\hline $\begin{array}{l}\text { Mujeres nulíparas con embarazo simple } \\
\text { en cefálica mayor o igual a } 37 \text { semanas, } \\
\text { se les realizó inducción del trabajo de } \\
\text { parto, o cesárea antes del inicio del } \\
\text { trabajo de parto. }\end{array}$ & $405(34,0)$ & $94 / 405(23,3)$ & $94 / 440(21,4)$ \\
\hline $\begin{array}{l}\text { Mujeres multíparas sin cicatriz uterina } \\
\text { previa, con un embarazo simple en } \\
\text { cefálica mayor o igual de } 37 \text { semanas y } \\
\text { trabajo de parto espontáneo. }\end{array}$ & $178(15,0)$ & $17 / 178(9,5)$ & $17 / 440(3,9)$ \\
\hline $\begin{array}{l}\text { Mujeres multíparas sin cicatriz uterina } \\
\text { previa, con un embarazo simple en } \\
\text { cefálica mayor o igual a } 37 \text { semanas, } \\
\text { a quienes se les realizó inducción del } \\
\text { trabajo de parto o cesárea antes del } \\
\text { inicio del trabajo de parto. }\end{array}$ & $118(9,9)$ & $21 / 118(17,7)$ & $21 / 440(4,8)$ \\
\hline $\begin{array}{l}\text { Todas las mujeres multíparas con } \\
\text { antecedente de al menos una cicatriz } \\
\text { uterina previa con embarazo mayor o } \\
\text { igual a } 37 \text { semanas, en cefálica. }\end{array}$ & $160(13,4)$ & $160 / 160(100)$ & $160 / 440(36,4)$ \\
\hline $\begin{array}{l}\text { Todas las mujeres nulíparas con embarazo } \\
\text { simple, en presentación podálica. }\end{array}$ & $10(0,8)$ & $10 / 10(100)$ & $10 / 440(2,3)$ \\
\hline $\begin{array}{l}\text { Todas las mujeres multíparas con } \\
\text { embarazo simple, en presentación } \\
\text { podálica, con o sin cicatriz uterina previa. }\end{array}$ & $12(1,0)$ & $12 / 12(100)$ & $12 / 440(2,7)$ \\
\hline $\begin{array}{l}\text { Todas las mujeres con embarazos } \\
\text { múltiples, con o sin cicatriz uterina } \\
\text { previa. }\end{array}$ & $9(0,8)$ & 9/9 (100) & $9 / 440(2,1)$ \\
\hline $\begin{array}{l}\text { Todas las mujeres con embarazos simples, } \\
\text { en presentación transversa u oblicua, } \\
\text { con o sin antecedente de cicatriz uterina } \\
\text { previa. }\end{array}$ & $3(0,3)$ & $3 / 3(100)$ & $3 / 440(0,7)$ \\
\hline $\begin{array}{l}\text { Todas las mujeres con embarazo de } 36 \\
\text { semanas o menos, con o sin cicatriz } \\
\text { uterina previa. }\end{array}$ & $90(7,6)$ & $39 / 90(43,3)$ & $39 / 440(8,9)$ \\
\hline Total & $1190(100)$ & $440 / 1190(37,0)$ & $440 / 440(100)$ \\
\hline
\end{tabular}




\begin{tabular}{|c|c|c|}
\hline Indicación médica & $\mathbf{N}$ & $\%$ \\
\hline Cesárea previa & 122 & 27,7 \\
\hline Inducción fallida & 80 & 18,2 \\
\hline Sospecha estado fetal insatisfactorio & 47 & 10,7 \\
\hline Preeclampsia severa / Hipertensión gestacional & 37 & 8,4 \\
\hline Macrosomía fetal / Desproporción cefalopélvica & 32 & 7,3 \\
\hline Presentación anormal del feto & 27 & 6,1 \\
\hline Detención en la dilatación / Trabajo de parto de prolongado & 26 & 5,9 \\
\hline Ruptura prolongada / Prematura de membranas / Corioamnionitis & 16 & 3,6 \\
\hline Placenta previa / abruptio placentae / hemorragia tercer trimestre & 15 & 3,4 \\
\hline Expulsivo prolongado & 13 & 3,0 \\
\hline Restricción de crecimiento intrauterino & 10 & 2,3 \\
\hline Embarazo múltiple & 9 & 2,0 \\
\hline Cervix desfavorable & 3 & 0,7 \\
\hline Herpes genital & 3 & 0,7 \\
\hline Total general & 440 & 100 \\
\hline
\end{tabular}

las cifras reportadas del aumento de cesáreas en el mundo entre los años 2005 y 2013 (1-3).

Los resultados del presente estudio son similares a investigaciones publicadas en la literatura internacional que también reportan que los grupos 2 y 5 aportan hasta dos terceras partes de la proporción de cesáreas realizadas en instituciones de diferentes niveles de complejidad (23, 31-33). Por otro lado, un estudio realizado en seis estados de Canadá, publicado en el 2013 (26), encontró que el grupo 5 representó el $11 \%$ del total de cesáreas, con una proporción específica del $89 \%$, seguido por el grupo 2 con una contribución del 13,1\% al total de cesáreas y una proporción específica de 44,6\%, que evidencia diferencias con respecto al presente estudio, tanto en el aporte de estos grupos a la proporción global de cesáreas, que fue de 36,4 y 21,4\%, respectivamente, como en la proporción específica encontrada de 100 y 23,3\% en el mismo orden. Esta marcada divergencia de resultados entre las diferentes instituciones refuerza la necesidad de contar con un diagnóstico específico de la institución antes de iniciar los procesos de intervención. El aporte a la proporción global de cesáreas por el grupo 5 podría ser modificado si se establece un protocolo de atención de parto vaginal después de cesárea (34), porque en ausencia de la implementación de dichos protocolos se debe esperar un incremento continuo y permanente en la proporción global de cesáreas dentro de las instituciones $(34,35)$.

En el presente estudio se encontró una relación entre la proporción de cesáreas entre los grupos 1 y 2 (36,6 y 23,3\% respectivamente) que equivale a 1,5:1. Esta relación es inferior a la razón teórica esperada según Robson y Hartigan, que debe ser mayor de 2:1 (32). Esto refleja que un número muy importante de mujeres son llevadas a cesárea en ausencia de trabajo de parto o luego de partos indu- 
cidos. Dado que la proporción específica de cesárea en el grupo 1 (36,6\%) es muy superior a la esperada según Robson y Hartigan, de 10 a $15 \%$, es necesario que para el grupo de mujeres nulíparas en presentación cefálica a término se realice un análisis dirigido a determinar la pertinencia de las indicaciones de cesárea (36). En este mismo sentido, la frecuencia de utilización de cesárea de 9,5\% encontrada en el grupo 3, superior al $3 \%$ recomendado, invita a una revisión de las indicaciones y conductas médicas en la institución $(32,36)$.

Existe acuerdo sobre los beneficios neonatales de la cesárea para las mujeres con feto en presentación podálica (grupos 6 y 7), las mujeres con embarazo gemelar cuando el primer feto se encuentra en presentación diferente a la cefálica (grupo 8) y las mujeres que presentan fetos en situación transversa (grupo 9), con tasa de cesárea para estos grupos de $100 \%(8,9,37)$. En conjunto, estos grupos constituyeron el 2,9\% del total de los nacimientos ocurridos y aportaron el 7,8\% de las cesáreas realizadas en la institución. Comparado con los reportes de British Columbia para 2010 y 2011, estos mismos grupos aportaron un 6\% de los nacimientos ocurridos y una participación del 16,5\% a la proporción global de cesáreas, revelando que para la institución la intervención sobre estos grupos mediante protocolos de versión cefálica externa o parto vaginal en pelvis tendría bajo impacto en dicha proporción (36).

Específicamente en Colombia, la clasificación de Robson se aplicó en el Hospital Universitario de San Vicente Fundación de Medellín. En esa institución, la mayor proporción de cesáreas se presentó en los grupos del 1 al 4 con 35; 55,4; 15 y $49 \%$ respectivamente, mientras que en el presente estudio las proporciones de cesárea específicas para estos mismos grupos fueron 36,6; 23,2; 9,5 y 17,7\% respectivamente. En ambos estudios se encuentra que las mujeres en trabajo de parto hacen una importante contribución a la proporción global de cesáreas. Sin embargo, la proporción de mujeres nulíparas en quienes se realizó cesárea luego de inducción o cesárea sin trabajo de parto $(23,6 \%)$ contrastan de manera favorable con lo reportado en los estudios realizados en Medellín y en el estudio de Ferreira et al. en Brasil, en los que la proporción de cesáreas para estos mismos grupos fue de 73 y $55,4 \%$ respectivamente (38). En ambos estudios, la contribución de estos grupos de mujeres incluidas en la clasificación de Robson a la proporción global de cesáreas es similar a la de la presente investigación, lo que hace que las proporciones reportadas para estas instituciones sean comparables, y sustenta que intervenciones dirigidas a disminuir el uso de la cesárea en estos grupos tendrían un impacto menor en el Hospital de Engativá que si se implementan en las instituciones referenciadas en Medellín y Brasil (19, 38).

El grupo 10, que corresponde a los nacimientos prematuros, es heterogéneo ya que se incluyen los trabajos de parto espontáneos, inducidos o las cesáreas realizadas antes del término, sin que se tenga clara la indicación de realizar el procedimiento como, por ejemplo, el peso fetal estimado de estas gestaciones (38-40). Se encontró que la tasa de cesárea en este grupo de gestantes fue del $43 \%$, mientras que para el estudio realizado en el Hospital de San Vicente fue del 34,9\% (19). El tamaño de este grupo (7,6\%) refleja que la institución es centro de referencia para casos muy seleccionados de parto prematuro, y la proporción de cesárea mayor al $40 \%$ indica que se están atendiendo mujeres con condiciones clínicas que obligan a hacer una terminación rápida y temprana del embarazo. Sin embargo, y a pesar de que este grupo contribuye solo con el 8,9\% de la proporción total de cesáreas, la maduración cervical y la monitorización electrónica fetal continua, pertinentes según la condición clínica materno-fetal, podrían disminuir la proporción específica de cesáreas dentro de este grupo particular (32).

Dentro de las fortalezas de este estudio se encuentra que se realizó la revisión de una muestra 
aleatoria cercana al $20 \%$ de los nacimientos ocurridos durante un periodo de tres años, que refleja adecuadamente la distribución de la población de mujeres atendidas en la institución. Asimismo, la verificación de la calidad de la información de la historia por duplicado y la validación de la base de datos con la clasificación realizada, disminuyen el riesgo de selección y de mala clasificación. No obstante, dado el carácter retrospectivo del estudio, no es posible determinar si hubo cambios en las conductas del personal de salud a lo largo del tiempo evaluado. En ese mismo sentido, otra limitación del estudio radica en que la indicación médica de las inducciones del parto y de las cesáreas no se documenta de manera estandarizada en los registros clínicos de las pacientes, lo que hizo imposible discriminar las indicaciones médicas de la cesárea para cada uno de los grupos de Robson y, por tanto, realizar el análisis detallado en los grupos en los que se encontraron proporciones específicas de cesárea elevadas.

Con base en los resultados obtenidos y las limitaciones del presente estudio, se propone que para una implementación efectiva del modelo en las instituciones es conveniente clasificar de manera prospectiva los nacimientos en cada uno de los grupos descritos y estandarizar la denominación de las indicaciones para inducción del trabajo de parto, la descripción de la indicación obstétrica o perinatal de la cesárea con especial énfasis en los partos pretérmino, las indicaciones y los protocolos de inducción y conducción del trabajo de parto, la definición de inducción fallida, el uso de partogramas y de los métodos de vigilancia del bienestar fetal, así como el reporte uniforme de los mismos, y los criterios y las indicaciones para realizar cesárea electiva anteparto. Esta información permitirá adecuar las estrategias e identificar intervenciones para racionalizar el uso de la cesárea como vía del parto $(35,37)$ y alcanzar metas cercanas al $15 \%$ según la propuesta de la OMS $(4,5,21)$ o al 20\%, que demuestran beneficio para el binomio madrehijo (41).

\section{CONCLUSIONES}

La clasificación Robson en un modelo aplicable en las instituciones de salud que permite identificar los grupos de mujeres que más contribuyen a la utilización de cesárea como vía del parto.

En la institución evaluada, los grupos de mujeres con tasas específicas más elevadas de cesárea son las mujeres con cesárea anterior, las nulíparas, y especialmente las sometidas a inducción del parto o cesárea electiva y las mujeres con embarazos pretérmino. Estos grupos podrían ser susceptibles de intervención para reducir la utilización de cesárea en cada uno de ellos, con un importante impacto sobre la proporción global de cesáreas de la institución.

\section{REFERENCIAS}

1. Rubio-Romero JA, Fonseca-Pérez JE, Molina S, Buitrago Leal M, Zuleta JJ, Ángel-Müller E, et al. Racionalización del uso de la cesárea en Colombia. Consenso de la Federación Colombiana de Obstetricia y Ginecología (Fecolsog) y la Federación Colombiana de Perinatología (Fecopen). Bogotá, 2014. Rev Colomb Obstet Ginecol. 2014;65:139-51.

2. Sistema Integral de Información de la Protección Social - Observatorio de salud materna de Colombia [Internet]. 2015 [visitado 2016 Feb 15]. Disponible en: http://www.sispro.gov.co/Pages/Observatorios/ salud_materna.aspx

3. Souza J, Betran A, Dumont A, de Mucio B, Gibbs Pickens C, Deneux-Tharaux C, et al. A global reference for caesarean section rates (C-Model): a multicountry cross-sectional study. BJOG. 2016;123:427-36.

4. World Health Organization. Appropriate technology for birth. Lancet Lond Engl. 1985;2:436-7.

5. Cyr RM. Myth of the ideal cesarean section rate: commentary and historic perspective. Am J Obstet Gynecol. 2006;194:932-6.

6. Villar J, Valladares E, Wojdyla D, Zavaleta N, Carroli G, Velazco A, et al. Caesarean delivery rates and pregnancy outcomes: the 2005 WHO global survey on maternal and perinatal health in Latin America. Lancet. 2006;367:1819-29. 
7. Lumbiganon P, Laopaiboon M, Gülmezoglu AM, Souza JP, Taneepanichskul S, Ruyan P, et al. Method of delivery and pregnancy outcomes in Asia: the WHO global survey on maternal and perinatal health 2007 08. Lancet Lond Engl. 2010;375:490-9.

8. Villar J, Carroli G, Zavaleta N, Donner A, Wojdyla D, Faundes A, et al. Maternal and neonatal individual risks and benefits associated with caesarean delivery: multicentre prospective study. BMJ. 2007;335:1025.

9. Liu S, Liston RM, Joseph KS, Heaman M, Sauve R, Kramer MS, et al. Maternal mortality and severe morbidity associated with low-risk planned cesarean delivery versus planned vaginal delivery at term. CMAJ. 2007;176:455-60.

10. Betrán AP, Gulmezoglu AM, Robson M, Merialdi M, Souza JP, Wojdyla D, et al. WHO global survey on maternal and perinatal health in Latin America: classifying caesarean sections. Reprod Health. 2009; $6: 1-8$.

11. Souza JP, Gülmezoglu A, Lumbiganon P, Laopaiboon M, Carroli G, Fawole B, et al. Caesarean section without medical indications is associated with an increased risk of adverse short-term maternal outcomes: the 2004-2008 WHO Global Survey on Maternal and Perinatal Health. BMC Med. 2010;8:71.

12. van Dillen J, Diesch M, Schutte J, Zwart J, Wolterbeek $\mathrm{R}$, van Roosmalen J. Comparing grades of urgency for classification of cesarean delivery. Int J Gynaecol Obstet. 2009; 107:16-8.

13. Althabe F, Belizán JM, Villar J, Alexander S, Bergel E, Ramos S, et al. Mandatory second opinion to reduce rates of unnecessary caesarean sections in Latin America: a cluster randomised controlled trial. Lancet Lond Engl. 2004;363:1934-40.

14. Anderson GM, Lomas J. Determinants of the increasing cesarean birth rate. Ontario data 1979 to 1982 . N Engl J Med. 1984;311:887-92.

15. Robson M. Classification of caesarean sections. Fetal Matern Med Rev. 2001;12:23-39.

16. Robson MS, Scudamore IW, Walsh SM. Using the medical audit cycle to reduce cesarean section rates. Am J Obstet Gynecol. 1996;174:199-205.
17. Robson MS. Can we reduce the caesarean section rate? Best Pract Res Clin Obstet Gynaecol. 2001;15: 179-94.

18. Brennan DJ, Robson MS, Murphy M, O’Herlihy C. Comparative analysis of international cesarean delivery rates using 10-group classification identifies significant variation in spontaneous labor. Am J Obstet Gynecol. 2009;201:308.e1-308.e8.

19. Zuleta-Tobón JJ, Quintero-Rincón F, QuicenoCeballos AM. Aplicación del modelo de Robson para caracterizar la realización de cesáreas en una institución de tercer nivel de atención en Medellín, Colombia: estudio de corte transversal. Rev Colomb Obstet Ginecol. 2013;64:90-9.

20. Betrán AP, Vindevoghel N, Souza JP, Gülmezoglu AM, Torloni MR. A systematic review of the Robson classification for caesarean section: what works, doesn't work and how to improve it. PloS One. 2014;9:e97769.

21. Lamy C, Alexander S. [The World Health Organisation proposes adopting the Robson Classification as an internationally applicable cesarean section classification system]. J Gynécologie Obstétrique Biol Reprod. 2015;44:587-90.

22. Chong C, Su LL, Biswas A. Changing trends of cesarean section births by the Robson Ten Group Classification in a tertiary teaching hospital. Acta Obstet Gynecol Scand. 2012;91:1422-7.

23. Vera GC, Correa BR, Neira MJ, Rioseco RA, Poblete LA. Utilidad de la evaluación de 10 grupos clínicos obstétricos para la reducción de la tasa de cesárea en un hospital docente. Rev Chil Obstet Ginecol. 2004;69:219-26.

24. Kelly S, Sprague A, Fell DB, Murphy P, Aelicks N, Guo $\mathrm{Y}$, et al. Examining caesarean section rates in Canada using the Robson classification system. J Obstet Gynaecol Can. 2013;35:206-14.

25. Vengoechea PJC, Pérez AC, Betrán AP, Morey M del MM, Borges MMF, Alcácer PX, et al. Clasificación de cesáreas por Grupos de Robson en dos periodos comparativos en el Hospital de Manacor. Prog Obstet Ginecol. 2010;53:385-90. 
26. Litorp H, Kidanto HL, Nystrom L, Darj E, Essén B. Increasing caesarean section rates among low-risk groups: a panel study classifying deliveries according to Robson at a university hospital in Tanzania. BMC Pregnancy Childbirth. 2013;13:107.

27. Lee YYC, Roberts CL, Patterson JA, Simpson JM, Nicholl MC, Morris JM, et al. Unexplained variation in hospital caesarean section rates. Med J Aust. 2013;199:348-53.

28. Maso G, Piccoli M, Montico M, Monasta L, Ronfani L, Parolin S, et al. Interinstitutional variation of caesarean delivery rates according to indications in selected obstetric populations: a prospective multicenter study. BioMed Res Int. 2013;2013:786563.

29. Sørbye IK, Vangen S, Oneko O, Sundby J, Bergsjø P. Caesarean section among referred and self-referred birthing women: a cohort study from a tertiary hospital, northeastern Tanzania. BMC Pregnancy Childbirth. 2011;11:55.

30. Maso G, Alberico S, Monasta L, Ronfani L, Montico M, Businelli C, et al. The application of the Ten Group classification system (TGCS) in caesarean delivery case mix adjustment. A multicenter prospective study. PloS One. 2013;8:e62364.

31. Barčaite E, Kemekliene G, Railaite DR, Bartusevičius A, Maleckiene L, Nadišauskiene R. Cesarean section rates in Lithuania using Robson Ten Group Classification System. Med Kaunas Lith. 2015;51:280-5.

32. Robson M, Hartigan L, Murphy M. Methods of achieving and maintaining an appropriate caesarean section rate. Best Pract Res Clin Obstet Gynaecol. 2013;27:297-308.

33. McCarthy FP, Rigg L, Cady L, Cullinane F. A new way of looking at Caesarean section births. Aust N Z J Obstet Gynaecol. 2007;47:316-20.
34. Rozen G, Ugoni AM, Sheehan PM. A new perspective on VBAC: a retrospective cohort study. Women Birth J Aust Coll Midwives. 2011;24:3-9.

35. Chaillet N, Dumont A. Evidence-based strategies for reducing cesarean section rates: a meta-analysis. Birth Berkeley Calif. 2007;34:53-64.

36. Examining cesarean delivery rates in British Columbia using the Robson Ten Classification [Internet]. [Visitado 2016 Feb 29]. Disponible en: http://www. perinatalservicesbc.ca/Documents/Data-Surveillance ReportSurveillanceSpecialReportRobsonTenClassifica tionDec2011.pdf

37. American College of Obstetricians and Gynecologists (College), Society for Maternal-Fetal Medicine, Caughey AB, Cahill AG, Guise J-M, Rouse DJ. Safe prevention of the primary cesarean delivery. Am J Obstet Gynecol. 2014;210:179-93.

38. Ferreira EC, Pacagnella RC, Costa ML, Cecatti JG. The Robson ten-group classification system for appraising deliveries at a tertiary referral hospital in Brazil. Int J Gynecol Obstet. 2015;129:236-9.

39. Evidence-based strategies for implementing guidelines in obstetrics: a systematic review. - PubMed - NCBI [Internet]. [Visitado 2016 Feb 5]. Disponible en: http://www.ncbi.nlm.nih.gov/pubmed/17077251

40. Chaillet N, Dumont A. Evidence-based strategies for reducing cesarean section rates: a meta-analysis. Birth Berkeley Calif. 2007;34:53-64.

41. Molina G, Weiser TG, Lipsitz SR, Esquivel MM, UribeLeitz T, Azad T, et al. Relationship Between Cesarean Delivery Rate and Maternal and Neonatal Mortality. JAMA. 2015 1;314:2263-70. 